\title{
EPM ephemerides and relativity
}

\author{
E. V. Pitjeva \\ Institute of Applied astronomy RAS, \\ Kutuzov quay 10, 191187 St. Petersburg, Russia \\ email: evp@ipa.nw.ru
}

\begin{abstract}
In the seventies of the last century the EPM ephemerides (Ephemerides of Planets and the Moon) of IAA RAS originated and have been developed since that time. These ephemerides are based upon relativistic equations of motion of celestial bodies and light rays and upon relativistic time scales. The updated model of EPM2008 includes the new values of planet masses and other constants, the improved dynamical model with adding Trans-Neptunian Objects and the expanded database (1913-2008). More than 260 parameters have been determined while improving the planetary part of EPM2008 to 550000 observations. EPM2008 have been oriented to ICRF by including into the total solution the VLBI data of spacecraft near the planets. The real uncertainty of EPM ephemerides has been checked by comparison with the JPL's DE ephemerides. Some estimates of the post-model parameters have been obtained:

$|1-\beta|<0.0002, \quad|1-\gamma|<0.0002, \dot{G} / G=(-5.9 \pm 4.4) \cdot 10^{-14}$ per year, the statistic zero corrections to the planet perihelion advances.
\end{abstract}

Keywords. Relativity, celestial mechanics, ephemerides, radar astronomy

\section{Historical introduction: general relativity in EPM ephemerides}

In the seventies of the last century to support space flights the EPM ephemerides (Ephemerides of Planets and the Moon) of IAA RAS originated at about the same time as DE ephemerides and have been developed since that time.

After the brilliant explanation by Einstein the strange $\left(43^{\prime \prime} / \mathrm{cy}\right)$ discrepancy between theoretical predictions and observations of the secular motion of Mercury perihelion, the planet ephemerides are to be constructed on the basis of General Relativity. The relativistic basis for constructing ephemerides was provided many years ago in the papers by Estabrook (1971), Will (1974), and it has been used for JPL (Standish, 1976), IAA RAS (Krasinsky et al., 1978), and MIT (Ash et al., 1967) ephemerides for more than 30 years. However, for the workers in Russia the main guide was the book of Brumberg (1972). Moreover, the relativistic equations of the planet motion may be given in different coordinate systems of the Schwarzschild metric (parameter $\alpha$ ), namely, standard, harmonic, izotropic, etc. However, planet coordinates turned out to be essentially different for the standard and harmonic systems. Brumberg (1979) proved that ephemeris construction and processing of observations should be done in the same coordinate system, in which case the dependence on the coordinate system (parameter $\alpha$ ) vanishes. Later on, the resolutions of IAU $(1991,2000)$ recommended to use harmonic coordinates for BCRS. Actually, harmonic coordinates have been used for all modern ephemerides since long ago.

Our first ephemerides of the inner planets which were analytical (Krasinsky et al., 1978) in contrast to more perfect analytical ephemerides of the Moon and planets by Chapront and Bretagnon were compared with optical and radar observations. Simultaneously we also computed numerical planet ephemerides. Our comparison revealed that numerical 
ephemerides were able to present accurate observations much better than any analytical theories did.

In the eighties of the previous century (for example, Krasinsky et al., 1986) we tested relativistic effects processing the observations available at that time. A purely Newtonian theory was developed and results were tested by both the relativistic and the Newtonian theories. It was proved that the relativistic ephemeris for any observed planets provides considerably better fit of the observations (by 10\%) than the Newtonian theory even if latter incorporates the observed perihelion secular motions. Moreover, at that time attempts to estimate PPN parameters $\beta, \gamma$ and the rate of changing of gravitation constant $\dot{G} / G$ were also made.

All the modern ephemerides: DE - JPL (Folkner et al., 2008), EPM - IAA RAS (Pitjeva, 2009), INPOP - IMCCE (Fienga et al., 2008) are based upon relativistic equations of motion for celestial bodies and light rays as well as relativistic time scales. The numerical integration of the equations of celestial bodies motion has been performed in the Parameterized Post-Newtonian metric for General Relativity in the TDB time scale; the relativistic effects of the signal delay (the Shapiro effect), and path-bending of the radio-signal propagation in the gravitation field of the Sun, Jupiter, Saturn and the reduction of observations from the proper time of the observer to the coordinate time of the ephemerides are taken into account while processing observations.

\section{Present EPM2008 ephemerides}

EPM ephemerides are computed by numerical integration of the equations of celestial bodies motion in the barycentric coordinate frame of J2000.0 by Everhart (1974) method over the 400 years interval (1800-2200) using the program package ERA-7 (ERA: Ephemeris Research in Astronomy) developed to support scientific research in dynamical and ephemeris astronomy (Krasinsky \& Vasilyev, 1997). This paper concerns a planet part of the EPM ephemerides; the group of George Krasinsky is now developing a lunar part of the EPM ephemerides and fitting it to the LLR data (Yagudina, 2009).

The mass values of the planets have been taken from the recent best determinations by different authors obtained from the data of spacecraft orbiting and passing near planets or from the observations of satellites of these planets (http://maia.usno.navy.mil/NSFA/ CBE.html). All other constants have been obtained inside the EPM2008 ephemeris fitting process.

The updated model of EPM2008 includes Eris (which surpasses Pluto in the mass) and the other 20 largest Trans-Neptunian Objects (TNO) into the process of the simultaneous numerical integration in addition to nine planets, the Sun, 301 biggest asteroids, the Moon as well as the lunar physical libration, and takes into account perturbations due to the solar oblateness and perturbation from the massive ring of small asteroids.

Moreover, some tests have been made for estimating the effect of other TNO on the motion of planets. Their perturbations have been modeled by the perturbation from a circular ring having a radius of $43 \mathrm{AU}$ and the five versions of different masses. The minimum mass of this ring is equal to the mass of 100000 bodies with $100 \mathrm{~km}$ in diameter and density is equal to $2 \mathrm{~g} / \mathrm{cm}^{3}$, it amounts to 110 masses of Ceres. The maximum mass of the ring is expected to be 100 times the minimum mass. Other test versions of the TNO ring surpass the minimum mass by 25,50 , and 75 times. The effect of the ring is only noticeable for more accurate observations - the spacecraft data, especially for ones from spacecraft near Jupiter and Saturn. The rms residuals and the weight unit errors for the data after fitting the standard and test EPM ephemerides have shown that all the test masses of the TNO ring except the minimum mass are too large and make the 
Table 1. Mean values and rms residuals for radiometric observations.

\begin{tabular}{l|l|r|r|r|c}
\hline \multicolumn{1}{c}{ Planet } & \multicolumn{1}{|c}{ Type of data } & Time interval & $\mathrm{N}$ & $\langle O-C\rangle$ & $\sigma$ \\
\hline MERCURY & $\tau[\mathrm{m}]$ & $1964-1997$ & 746 & 0 & 575 \\
VENUS & $\tau[\mathrm{m}]$ & $1961-1995$ & 1354 & -2 & 584 \\
& Magellan $d r[\mathrm{~mm} / \mathrm{s}]$ & $1992-1994$ & 195 & 0 & 0.007 \\
& MGN,VEX VLBI [mas] & $1990-2007$ & 22 & 1.6 & 3.0 \\
& Cassini $\tau[\mathrm{m}]$ & $1998-1999$ & 2 & 4.0 & 2.4 \\
MEX $\tau[\mathrm{m}]$ & $2006-2007$ & 547 & 0.0 & 2.6 \\
& $\tau[\mathrm{m}]$ & $1965-1995$ & 403 & 0 & 719 \\
& Viking $\tau[\mathrm{m}]$ & $1976-1982$ & 1258 & 0 & 8.8 \\
& Viking $d \tau[\mathrm{mm} / \mathrm{s}]$ & $1976-1978$ & 14978 & -0.02 & 0.89 \\
& Pathfinder $\tau[\mathrm{m}]$ & 1997 & 90 & 0 & 2.8 \\
& Pathfinder $d \tau[\mathrm{mm} / \mathrm{s}]$ & 1997 & 7569 & 0 & 0.09 \\
& MGS $\tau[\mathrm{m}]$ & $1998-2006$ & 7342 & 0 & 1.4 \\
& Odyssey $\tau[\mathrm{m}]$ & $2002-2008$ & 5257 & 0 & 1.2 \\
& MRO $\tau[\mathrm{m}]$ & $2006-2007$ & 380 & 0 & 2.5 \\
JUPITER & spacecraft VLBI $[\mathrm{mas}]$ & $1984-2007$ & 96 & 0.0 & 0.7 \\
& spacecraft $\tau[\mathrm{m}]$ & $1973-2000$ & 7 & 0.0 & 11.8 \\
SATURN & spacecraft VLBI $[\mathrm{mas}]$ & $1996-1997$ & 24 & -1.8 & 9.5 \\
URANUS & spacecraft $\tau[\mathrm{m}]$ & $1979-2006$ & 33 & 1.0 & 20.2 \\
NEPTUNE & Voyager-2 $\tau[\mathrm{m}]$ & 1986 & 1 & 1.9 & 105 \\
\hline
\end{tabular}

Notes: VEX, MGS, Odyssey, MRO data are normal points representing about 400000 original observations.

data residuals worse. Thus, the upper limit of the mass of the TNO ring $\left(5.26 \cdot 10^{-8} \mathrm{M}_{\odot}\right)$ has been obtained.

Database, to which EPM2008 have been adjusted includes (in addition to previous observations since 1913) the recent spacecraft measurements, namely, ranging to Venus Express (VEX), Odyssey, Mars Reconnaissance Orbiter (MRO) and VLBI data of Odyssey and MRO (2006-2008), three-dimensional normal point observations of Cassini (20042006), along with CCD Flagstaff and TMO data of the outer planets and their satellites (2006-2008). These measurements have resulted in a significant improvement of planet orbits, especially for Venus and Saturn and the orientation of the EPM2008 ephemerides to ICRF. The most part of observations has been taken from the database of the IAU Commission 4 created by Myles Standish and continuing by William Folkner.

About 260 parameters have been determined while improving the planetary part of EPM2008 to more than 550000 data:

- the orbital elements of all the planets and 18 satellites of the outer planets observations those have been used to improve the orbits of these planets;

- the value of the Astronomical Unit in m;

- three orientation angles of the ephemerides relative to the International Celestial Reference Frame (ICRF) and their velocities;

- 13 rotation parameters of Mars and the coordinates of the three landers on the martian surface;

- masses of the ten asteroids that perturb Mars most strongly, mean densities for three taxonomic classes of asteroids ( $\mathrm{C}, \mathrm{S}, \mathrm{M})$, the mass and the radius of the asteroid ring, the ratio masses of the Earth and the Moon;

- the solar quadrupole moment $\left(J_{2}\right)$ and 21 parameters of the solar corona for different conjunctions with the Sun;

- eight coefficients of Mercury's topography and the corrections to the surface levels of Venus and Mars;

- five coefficients of the phase effect correction for the outer planets;

- constant bias for spacecraft and some radar planet observations, that were interpreted as calibration errors of the instruments or as systematic errors of unknown origin; 

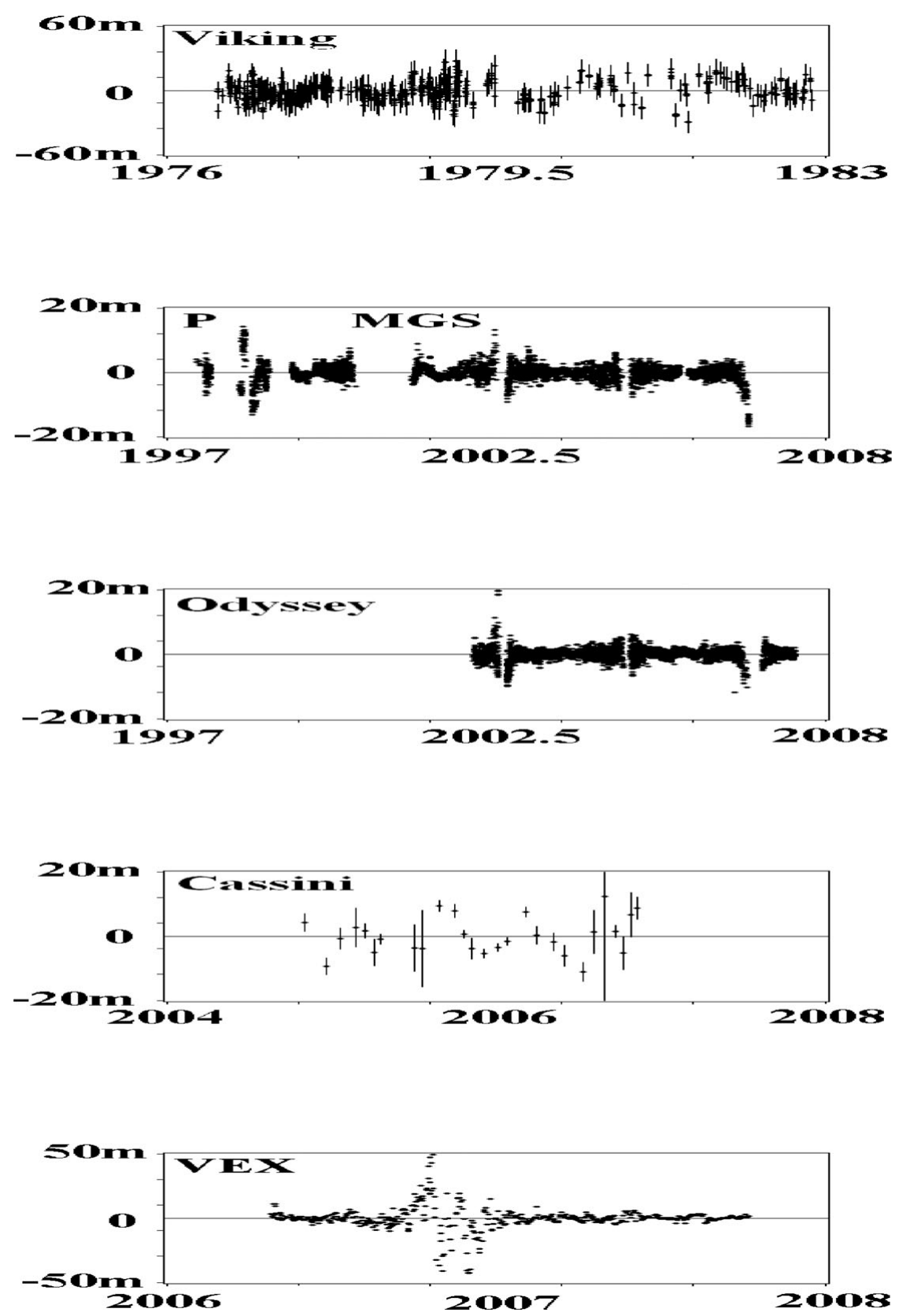

Figure 1. Viking, Pathfinder (P), MGS, Odyssey, Cassini, VEX range residuals

- the post-model parameters $(\beta, \gamma, \dot{G} / G$, secular trends of the planet perihelia and semi-major axes).

Mean values and rms residuals of observations are presented in Tables 1, 2 and on Fig. 1, 2. The data residuals don't exceed their a priori accuracies. The rms residuals of ranging for Viking are $8.8 \mathrm{~m}$, for Pathfinder $2.8 \mathrm{~m}$, for MGS and Odyssey $1.2-1.4 \mathrm{~m}$, for Cassini (Saturn) $3.0 \mathrm{~m}$, for VEX $2.6 \mathrm{~m}$. 

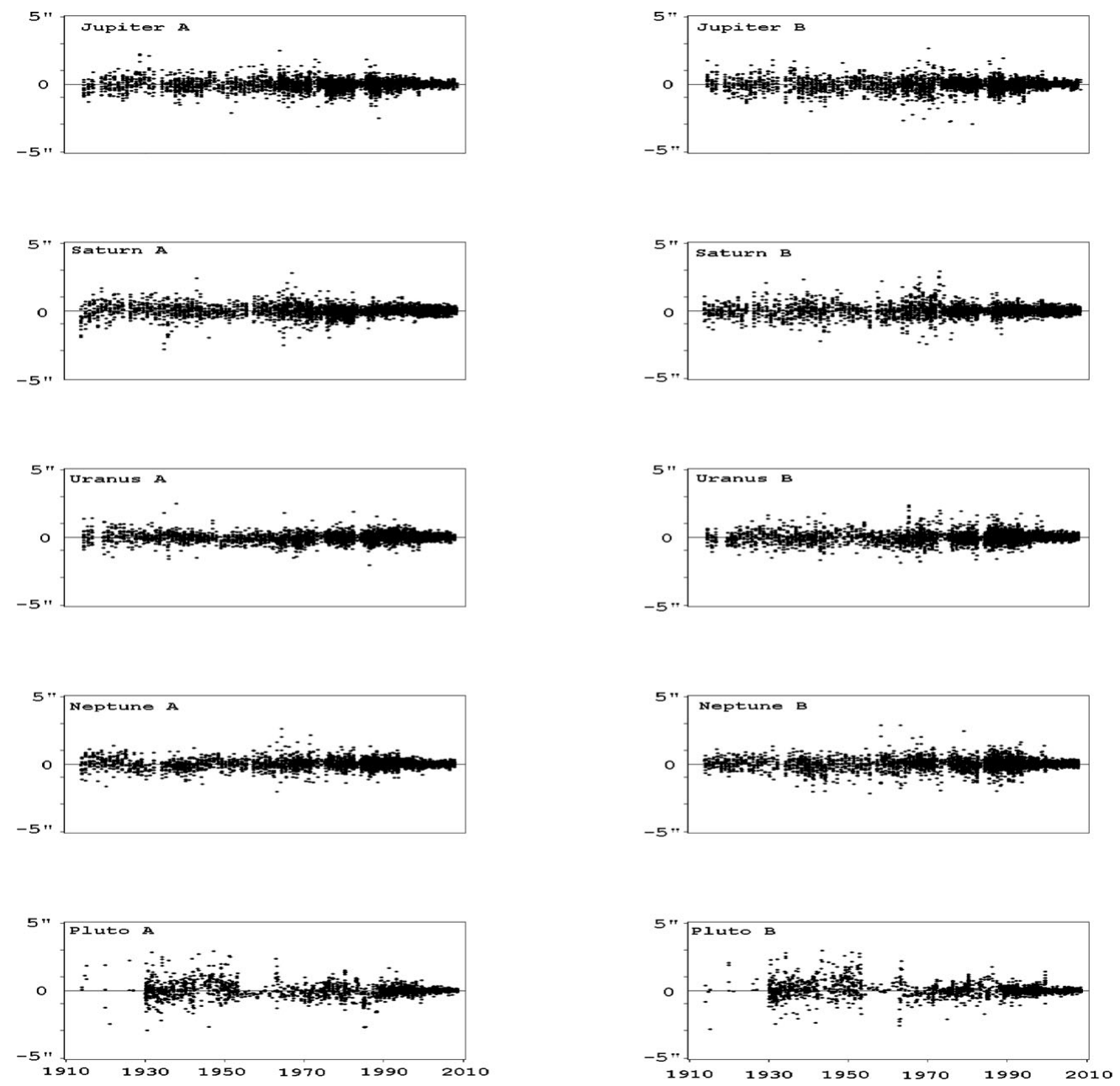

Figure 2. Residuals of the outer planets 1913-2008 in $\alpha \cos \delta(\mathrm{A})$ and in $\delta(\mathrm{B})$, the scale $\pm 5^{\prime \prime}$.

EPM2008 have been oriented to ICRF by including into the total solution the 118 ICRF-base VLBI measurements of spacecraft (Magellan, Phobos, MGS, Odyssey, VEX, and MRO) 1989-2007 near Venus and Mars. Several solutions for recent and previous data are given in Table 3.

The obtained values of the Astronomical Unit, the Moon-Earth mass ratio

$\mathrm{AU}=(149597870697 \pm 3) \mathrm{m}, M_{\text {Earth }} / M_{\text {Moon }}=81.3005676 \pm 0.0000030$

and masses of several asteroids (Table 4) are presented with their real uncertainties estimated by comparing the values obtained in dozens of different test LS solutions that differed by the sets of observations, their weights, and the sets of parameters included in the solution, as well as by comparing parameter values produced by independent groups. The discussion of these values and their comparison with values obtained by other authors are given in the paper by Pitjeva \& Standish (2009).

\section{Comparison of DE and EPM ephemerides}

The differences between various ephemerides are useful to know since they are indicative of the realistic accuracies of the ephemerides. The comparison of our recent EPM2008 
Table 2. Mean values and rms residuals for optical observations and spacecraft encounters* $\alpha$ and $\delta$ in mas, $1913-2008$.

\begin{tabular}{|c|c|c|c|c|c|}
\hline Planet & $\mathrm{N}$ & $\mid<O-C>_{\alpha}$ & $\sigma_{\alpha}$ & $\mid<O-C>_{\delta}$ & $\sigma_{\delta}$ \\
\hline VENUS* & 4 & 1.5 & 2.0 & 1 & 6.5 \\
\hline JUPITER & 12518 & 15 & 187 & -30 & 199 \\
\hline JUPITER* & 16 & 0.1 & 1.9 & -4.1 & 6.1 \\
\hline SATURN & 14296 & -1 & 167 & -3 & 160 \\
\hline SATURN* & 68 & 2.2 & 2.9 & 4.2 & 5.9 \\
\hline URANUS & 11446 & 6 & 178 & 2 & 208 \\
\hline URANUS* & 2 & -45 & 9 & -25 & 12 \\
\hline NEPTUNE & 10982 & 7 & 160 & 9 & 205 \\
\hline NEPTUNE* & 2 & -11 & 3.5 & -14 & 4.0 \\
\hline PLUTO & 5134 & 1 & 191 & 6 & 197 \\
\hline
\end{tabular}

Table 3. The rotation angles for the orientation of EPM onto ICRF.

\begin{tabular}{c|c|c|c|c}
\hline $\begin{array}{c}\text { Time } \\
\text { interval }\end{array}$ & $\begin{array}{c}\text { Number } \\
\text { of obs. }\end{array}$ & $\begin{array}{c}\varepsilon_{\mathrm{x}} \\
\text { mas }\end{array}$ & $\begin{array}{c}\varepsilon_{\mathrm{y}} \\
\text { mas }\end{array}$ & $\begin{array}{c}\varepsilon_{\mathrm{z}} \\
\text { mas }\end{array}$ \\
\hline $1989-1994$ & 20 & $4.5 \pm 0.8$ & $-0.8 \pm 0.6$ & $-0.6 \pm 0.4$ \\
$1989-2003$ & 62 & $1.9 \pm 0.1$ & $-0.5 \pm 0.2$ & $-1.5 \pm 0.1$ \\
$1989-2007$ & 118 & $-1.53 \pm 0.06$ & $1.02 \pm 0.06$ & $1.27 \pm 0.05$ \\
\hline
\end{tabular}

Table 4. Masses of Ceres, Pallas, Juno, Vesta, Iris, Bamberga in $\left(\mathrm{GM}_{\mathrm{i}} / \mathrm{GM}_{\odot}\right) \cdot 10^{-10}$.

\begin{tabular}{rrrrrr}
\hline (1) Ceres & (2) Pallas & (3) Juno & (4) Vesta & (7) Iris & (324) Bamberga \\
\hline 4.71 & 1.06 & 0.129 & 1.32 & 0.040 & 0.046 \\
\pm 0.03 & \pm 0.03 & \pm 0.008 & \pm 0.03 & \pm 0.008 & \pm 0.008 \\
\hline
\end{tabular}

ephemeris with the standard DE405 and the latest DE421 ephemerides has been made (Fig. 3, Table 5). The differences of heliocentric distances for the inner planets between EPM2008 and DE405 or DE421 are small. It is necessary to say about the real accuracy of DE405 ephemerides. Right now, 12 years after the DE405 construction and 27 years after observations of Viking-1 (with more accurate data included in this ephemeris) the residuals for modern data for Odyssey don't surpass $200 \mathrm{~m}$ (Konopliv et al., 2006), and as Fig. 3, Table 5 demonstrate it. It is evident that modeling the Mars motion is more difficult than other planets because of a large number of asteroids perturbing its orbit. The availability of a number of spacecraft near Jupiter and Saturn (besides optical observations) allows their ephemerides to be known better than those of other outer planets. The Fig. 3, Table 5 show the significant progress in agreement (and in reduction of uncertainties) of the orbits of all the planets especially owing to the VEX data (they were kindly given to us by Dr. Fienga) and the Cassini data.

\section{Relativistic tests and estimation of post-model parameters}

At present, the relativistic terms from the Sun and all the planets are included into the motion equations for integration. However, it is interest to estimate how relativistic terms from different planets influence modern planet observations. In addition to the basic EPM2008 (case 1) with the total account of all the relativistic terms in motion equations, three test ephemerides have been constructed:

a) without Saturn relativistic terms (case 2);

b) without Jupiter relativistic terms (case 3);

c) taking into account only solar relativistic terms (case 4).

Then all these ephemerides were improved to all 550000 observations of planets of different types (1913-2008). All the angular observations (both classical and modern CCD 
DE405-EPM2008
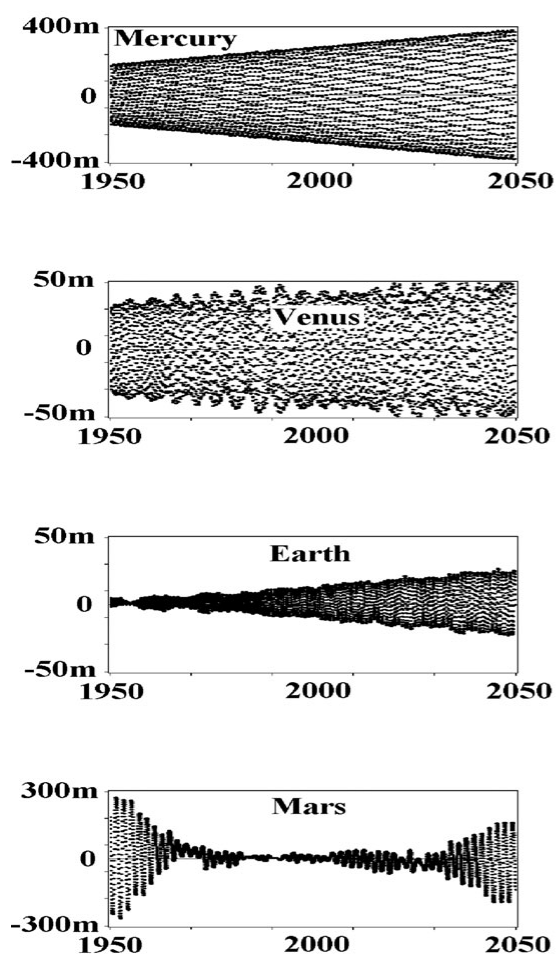

DE421-EPM2008
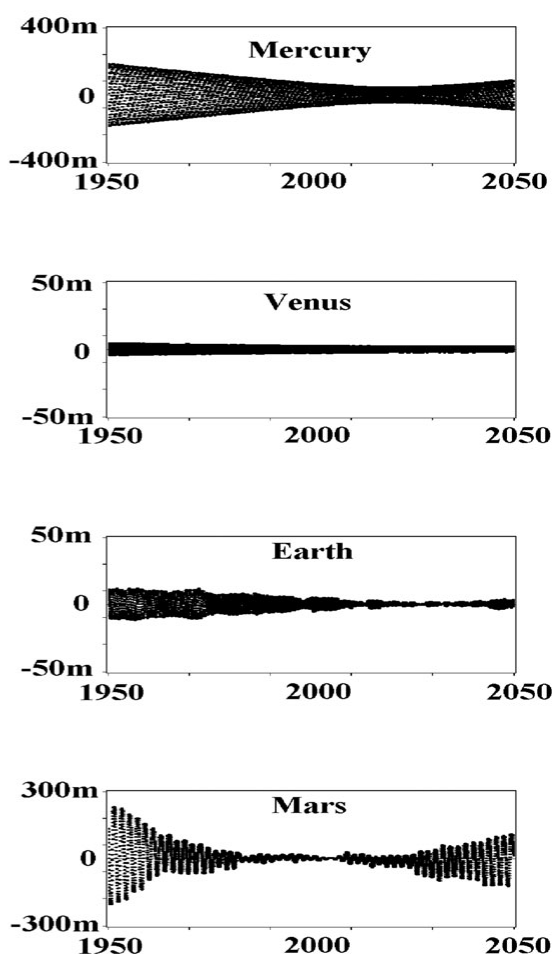

Figure 3. Differences in the heliocentric distances of inner planets for DE and EPM ephemerides, $1950-2050$.

Table 5. Maximum differences in the heliocentric distances of planets for DE and EPM ephemerides, $1950-2050$.

\begin{tabular}{l|c|c}
\hline Planet & DE405-EPM2008 & DE421-EPM2008 \\
\hline Mercury & $384 \mathrm{~m}$ & $185 \mathrm{~m}$ \\
Venus & $53.7 \mathrm{~m}$ & $4.6 \mathrm{~m}$ \\
Earth & $26.8 \mathrm{~m}$ & $11.9 \mathrm{~m}$ \\
Mars & $272 \mathrm{~m}$ & $233 \mathrm{~m}$ \\
Jupiter & $19.7 \mathrm{~km}$ & $4.8 \mathrm{~km}$ \\
Saturn & $29.3 \mathrm{~km}$ & $0.4 \mathrm{~km}$ \\
Uranus & $864 \mathrm{~km}$ & $310 \mathrm{~km}$ \\
Neptune & $6100 \mathrm{~km}$ & $848 \mathrm{~km}$ \\
Pluto & $29000 \mathrm{~km}$ & $1800 \mathrm{~km}$ \\
\hline
\end{tabular}

ones) and even VLBI data from spacecraft near planets don't show any differences in all cases. It is only the high-precision ranging that shows small differences. All the results are in given in Table 6 . The results demonstrate that Saturn terms (from the comparison of the cases 1 and 2) don't affect the residuals of the observations. Comparison of the 3 and 4 cases show that all the other planet terms (except Jupiter) don't have effects also. Actually, for modern planet observations it is necessary to take into account only relativistic terms from the Sun and Jupiter; moreover, before the appearance of the recent spacecraft ranging data with 1-2 $\mathrm{m}$ accuracy it had been possible to take into account only the solar relativistic terms without introducting any errors.

The high-accuracy modern observations not only made it possible to improve the orbital elements of planets and values related to the ephemerides but enable to determine 
Table 6. The rms residuals in $\mathrm{m}$ and the weight unit errors $\sigma_{0}$ for EPM ephemerides, accounting for different relativistic members.

\begin{tabular}{|c|c|c|c|c|c|c|}
\hline $\begin{array}{c}\text { Observations } \\
\text { Interval } \\
\text { Numbers n.p. }\end{array}$ & $\begin{array}{c}\text { planet } \\
\text { ranging } \\
1961-1997 \\
2504\end{array}$ & $\begin{array}{c}\text { Martian } \\
\text { landers } \\
1976-1997 \\
1348 \\
\end{array}$ & $\begin{array}{c}\text { Martian } \\
\text { spacecraft } \\
1998-2008 \\
13903 \\
\end{array}$ & $\begin{array}{c}\text { Venus } \\
\text { Express } \\
2006-2007 \\
547 \\
\end{array}$ & $\begin{array}{c}\text { Cassini } \\
\text { at Saturn } \\
2004-2006 \\
31 \\
\end{array}$ & $\begin{array}{c}1913-2008 \\
97101\end{array}$ \\
\hline All relat & 6 & 11.75 & 2.0 & 2.59 & 3.29 & 0.874 \\
\hline Without Saturn & 612.37 & 11.76 & 2.03 & 2.62 & 3.29 & 0.875 \\
\hline Without Jupiter & 613.14 & 11.95 & 2.36 & 3.74 & 5.24 & 0.916 \\
\hline Only Sun & 613.25 & 12.04 & 2.37 & 3.85 & 5.63 & 0.926 \\
\hline
\end{tabular}

Table 7. Variations of $\dot{G} / G$ and $\dot{a}_{i} / a_{i}=\mathrm{R}_{i}$ per year with $3 \sigma$ uncertainties.

\begin{tabular}{c|c|c|c|c|c}
\hline$\dot{G} / G \cdot 10^{-14}$ & $\mathrm{R}_{V e} \cdot 10^{-14}$ & $\mathrm{R}_{E a} \cdot 10^{-16}$ & $\mathrm{R}_{M a} \cdot 10^{-16}$ & $\mathrm{R}_{J u} \cdot 10^{-12}$ & $\mathrm{R}_{S a} \cdot 10^{-13}$ \\
\hline-5.87 & 8.99 & 1.36 & 2.36 & 9.14 & 6.74 \\
\pm 4.44 & \pm 8.73 & \pm 0.99 & \pm 1.65 & \pm 69.48 & \pm 50.73 \\
\hline
\end{tabular}

some small physical parameters characterizing the fundamental properties of our physical space. The EPM2008 ephemerides have been used to analyse these data. Unfortunately, the real accuracy of the parameters is reduced by order of magnitude or more because of systematic errors of observations of an unknown origin, impossibility to completely allow for the delay in the solar corona, and large correlations between parameters. However some estimations may be obtained, their real uncertainties were obtained from the comparison of many different versions of the solution. The PPN parameters and the quadrupole moment of the Sun $\left(J_{2}\right)$ producing various secular and periodic effects in orbital elements of planets have been estimated from the simultaneous solution:

$$
J_{2}=(2.0 \pm 0.5) \cdot 10^{-7},|\beta-1|<0.0002,|\gamma-1|<0.0002 .
$$

The variability of $G \dot{M}_{\odot} / G M_{\odot}$ should cause the corresponding variation of the semimajor axes of the planetary orbits. In this case the angular momentum integral holds: $G M_{\odot}(t) \cdot a(t)=$ const, then $G \dot{M}_{\odot} / G M_{\odot}=-\dot{a}_{\mathrm{i}} / a_{\mathrm{i}}$.

As Dr. Nikolay Pitjev has proposed an attempt to estimate these values. The values of variation of the semi-major axes of the planetary orbits are found stable and have a quite good accuracy for planets covered by the high-accurate data from spasecraft. The results obtained simultaneously for $\dot{G} / G$ and $\dot{a}_{\mathrm{i}} / a_{\mathrm{i}}$ per year of the semi-major axes with their $3 \sigma$ uncertainties are given in Table 7 . It is to be noted that all the semi-major axes of the planets are increasing while $\mathrm{GM}_{\odot}$ decreases (the Sun is losing its mass), as it should be. The average weighted value obtained from $\dot{a}_{\mathrm{i}} / a_{\mathrm{i}}$ is

$$
G \dot{M}_{\odot} / G M_{\odot}=(-1.63 \pm 1.50) \cdot 10^{-16} \text { per year. }
$$

This result is preliminary, it demands further improvement and discussion. The obtained value is significantly less than the adjusted $\dot{G} / G$ value (Table 7 ) and the supposed mass reduction of the Sun owing to the solar radiation and wind (of the order $-8 \cdot 10^{-14}$ /year). This discrepancy may to be part of the reason for comet falling on the Sun. The main result (Table 7) is

$$
\dot{G} / G=(-5.9 \pm 4.4) \cdot 10^{-14} \text { per year }(3 \sigma) .
$$

The corrections to the perihelion advances for the planets show to what extent the constructed model of the planet motion corresponds to the observations. In particular, the corrections for the inner planets demonstrate correspondence to General Relativity and to the value of the solar oblateness included into ephemerides. The corrections for the outer planets show agreement or non-agreement to the Newtonian theory of gravitation. 
Table 8. Corrections to the perihelion advances of planets $("$ / cy) and their real uncertainties.

\begin{tabular}{c|c|c|c|c}
\hline Mercury & Venus & Earth & Mars & Author \\
\hline 42.98 & 8.62 & 3.84 & 1.35 & Brumberg, 1972 \\
\hline $0.11 \pm 0.22$ & $-3.03 \pm 0.71$ & $-0.12 \pm 0.16$ & $-0.35 \pm 0.24$ & Pitjeva, 1986 \\
$-0.017 \pm 0.052$ & - & - & - & Pitjeva, 1993 \\
$-0.0040 \pm 0.0050$ & $0.024 \pm 0.033$ & $0.006 \pm 0.007$ & $-0.007 \pm 0.007$ & Pitjeva, 2009 \\
\hline Jupiter & Saturn & Uranus & Neptune & Pluto \\
\hline $0.067 \pm 0.093$ & $-0.010 \pm 0.015$ & $-3.89 \pm 3.90$ & $-4.44 \pm 5.40$ & $2.84 \pm 4.51$ \\
\hline
\end{tabular}

The obtained values (Table 8) are within the limits of their real uncertainties, in other words, the corrections to the planet perihelion advances are statistic zero.

\section{Conclusion}

Further improvement of the planet ephemerides and their parameters depends on the accuracy of modeling which results from the better knowledge of masses of celestial bodies including asteroids and TNO as well as decreasing errors of radiometrical data which originate from the ageing delay due to the solar corona and the spacecraft transponder.

\section{References}

Ash, M. E., Shapiro, I. I., \& Smith, W. B. 1967, AJ, 72, 332

Brumberg, V. A. 1972, in: V. G. Demin (ed.), Relativistic Celestial Mechanics (Moscow)

Brumberg, V. A. 1979, Celest. Mech. 20, 329

Estabrook, F. B. 1971, in: Derivation of Relativistic Lagrangian for n-Body Equations Containing Relativity Parameters $\beta$ and $\gamma$, JPL Internal Communication

Everhart, E. 1974, Celest. Mech., 10, 35

Fienga, A., Manche, H., Laskar, J., \& Gastineau, M. 2008, A\& A, 477, 315

Folkner, W. M., Williams, J. G., \& Boggs, D. H. 2008, Interoffice Memorandum, 343.R-08-003

Konopliv, A. S., Yoder, C. F., Standish, E. M., Yuan, D. N., \& Sjogren, W. L., 2006, Icarus, 182,23

Krasinsky, G. A., Pitjeva, E. V., Sveshnikov, M. L., \& Sveshnikova, E. S. 1978, Trudy Inst. Theoretical astronomy, 17, 46, in Russian.

Krasinsky, G. A., Aleshkina, E. Yu., Pitjeva, E. V., \& Sveshnikov, M. L. 1986, in: J. Kovalevsky, \& V. A. Brumberg (eds.), Relativity in Celestial Mechanics and Astrometry, Proc. IAU Symposium No. 114 (Dordrecht: D. Reidel Publ.Com.), p. 315

Krasinsky, G. A. \& Vasilyev, M. V. 1997, in I. M. Wytrzyszczak, J. H. Lieske \& R. A. Feldman (eds.), Dynamics and Astrometry of Natural and Artificial Celestial Bodies, Proc. IAU Colloquim No. 165 (Dordrecht: Kluwer Academic Publishers), p. 239

Pitjeva, E. V. 1986, Byull. Inst. T. A. Ross. Akad. Nauk, 15, 538, in Russian

Pitjeva, E. V. 1993, Celest. Mech. Dyn. Astr., 55, 313

Pitjeva, E. V. 2005, Astron. Letters, 31, 310

Pitjeva, E. V. 2009, in: M. Soffel \& N. Capitane (eds.), Astrometry, Geodynamics and Astronomical Reference Systems, Proc. JOURNEES-2008 (Dresden), p. 57

Pitjeva, E. V. \& Standish E. M. 2009, Celest. Mech. Dyn. Astr., 103, 365

Standish, E. M. Jr., Keesey, M. W., \& Newhall, XX 1976, Technical Report, JPL, 32-1603, 35 p.

Will, C. M. 1974, in: B. Bertotti (ed.), The Theoretical Tools of Experimental Gravitation, Experimental Gravitation (Academic Press)

Yagudina, E. I. 2009, in: M. Soffel \& N. Capitane (eds.), Astrometry, Geodynamics and Astronomical Reference Systems, Proc. JOURNEES-2008 (Dresden), p. 61 